Meta

Journal des tradlucteurs

Translators' Journal

\title{
La compréhension des énoncés spécialisés chez le traducteur : quelques réflexions
}

\section{Daniel Gile}

Volume 31, numéro 4, décembre 1986

URI : https://id.erudit.org/iderudit/002906ar

DOI : https://doi.org/10.7202/002906ar

Aller au sommaire du numéro

Éditeur(s)

Les Presses de l'Université de Montréal

ISSN

0026-0452 (imprimé)

1492-1421 (numérique)

Découvrir la revue

Citer cet article

Gile, D. (1986). La compréhension des énoncés spécialisés chez le traducteur : quelques réflexions. Meta, 31(4), 363-369. https://doi.org/10.7202/002906ar d'utilisation que vous pouvez consulter en ligne.

https://apropos.erudit.org/fr/usagers/politique-dutilisation/ 


\title{
LA COMPRÉHENSION DES ÉNONCÉS SPÉCIALISÉS CHEZ LE TRADUCTEUR : QUELQUES RÉFLEXIONS
}

\author{
DANIEL GILE \\ Traducteur-interprète, INALCO, Paris, France
}

\section{INTRODUCTION}

Le "mystère " de la compréhension du texte technique chez le traducteur et du discours technique chez l'interprète intrigue ceux qui utilisent leurs services ainsi que d'autres observateurs, ce qui se manifeste notamment à travers de fréquentes questions à ce sujet lors des conférences internationales. En général, deux positions prévalent parmi les observateurs : selon certains, pour traduire, il suffit de " connaitre les mots » sans les comprendre ; d'autres pensent qu'il faut comprendre pour traduire, et la compréhension étant une affaire de spécialistes, seuls les traducteurs spécialisés ayant euxmêmes une formation dans le domaine concerné sont capables de travailler correctement (voir par exemple Kourganoff 1980).

Les traducteurs et interprètes, pour leur part, affirment que la compréhension est nécessaire à la traduction. Le principe a été posé dès 1952 par Jean Herbert ; dans son classique Manuel de l'interprète, il affirme que :

avant de pouvoir interpréter un discours, il faut le comprendre aussi bien que possible (p. 10).

Seize ans plus tard, Danica Séleskovitch reprenait l'idée à son compte dans son livre l'Interprète dans les conférences internationales (1968), où elle précisait que la compréhension de l'interprète n'est pas aussi approfondie que celle d'un spécialiste, car ses connaissances sont inférieures, mais qu'elle lui permet de suivre le raisonnement du spécialiste :

on peut comprendre les nécessités d'une anastomose sans savoir opérer ni même décrire l'opération, mais il faut un minimum de connaissances anatomiques pour comprendre ce qui en est dit ; pour comprendre qu'un réacteur diverge lorsque son coefficient de relativité dépasse 1 , il faut posséder des connaissances certaines mais il n'est pas nécessaire d'être ingénieur atomiste; on peut suivre sans peine un raisonnement qui conclut à la nécessité d'ajouter des minéraux dans le fourrage du bétail, admettre le fait que azote et chlorure de potassium engraissent le sol, et être en mesure de suivre le raisonnement qui s'enchaîne sans être ingénieur agronome ou chimiste, etc. (p. 111)

Il semble maintenant acquis qu'il faut une certaine compréhension pour traduire. Nous pensons toutefois comme D. Séleskovitch, et à la différence d'autres auteurs (voir 
par exemple Folkart 1984), que cette compréhension ne doit pas nécessairement être aussi approfondie que celle du destinataire.

Le présent article vise à justifier cette prise de position et à examiner de plus près la nature de la " compréhension " qui permet à un traducteur de restituer dans une langue donnée des informations spécialisées formulées dans une autre langue et se situant dans un domaine qu'il connaît peu ou prou.

\section{POURQUOI FAUT-IL COMPRENDRE POUR TRADUIRE?}

L'énoncé est l'aboutissement d'un processus de " codage " d'un message dans une langue. S'il était possible de dégager $A$ et une deuxième langue $B$, la traduction pourrait se faire par simple application de ces règles et il ne serait nullement nécessaire de comprendre pour traduire. C'est d'ailleurs dans cette voie que s'étaient engagées dans les années 50 les premières tentatives de traduction automatique qui ont connu l'échec que l'on sait.

Or, les langues ne sont pas isomorphes, car elles désignent la réalité de manière polysémique et avec des structures stylistiques, syntaxiques et lexicales différentes et non parallèles. Comment établir par exemple des applications biunivoques entre la dichotomie française du tutoiement et du vouvoiement et le " you " anglais, entre les formes verbales en hébreu et en français ; comment concilier la flexibilité japonaise qui autorise les répétitions de mots avec l'interdit stylistique qui les frappe en français?

En outre, les polysémies ne sont pas nécessairement parallèles même là où un isomorphisme partiel existe. Il en résulte que si le transcodage peut donner des résultats acceptables dans des circonstances particulières (vocabulaire limité et monosémique, structures linguistiques limitées et prédéfinies), dans l'ensemble, il donne en langue d'arrivée des textes maladroits au mieux, et souvent incompréhensibles.

La traduction par transcodage ne donne donc qu'un projet de texte qui nécessite une révision. Celle-ci implique souvent un remaniement en profondeur de l'énoncé, dont le coût en temps et en efforts, pour une révision devant aboutir à une traduction de bonne qualité, peut dépasser sensiblement le coût de la traduction, comme le montre clairement l'expérience quotidienne des réviseurs.

Au transcodage, trop inefficace, les traducteurs professionnels préfèrent le « recodage "du message après le "décodage " de l'énoncé original : il s'agit non pas de passer directement de la langue de départ à la langue d'arrivée, mais d'extraire le message de l'énoncé original, puis de le reformuler en langue d'arrivée.

L'extraction du message à partir de l'énoncé en langue de départ implique une certaine " compréhension ». D'autre part, à l'évidence, le traducteur ne peut « comprendre " un énoncé spécialisé comme le destinataire, puisqu'il n'a pas la même base cognitive. Ces deux éléments sont à l'origine de l'idée d'une " compréhension " nécessaire et suffisante du traducteur, différente de celle du destinataire.

\section{L'ÉNONCÉ SCIENTIFIQUE ET TECHNIQUE}

La syntaxe

Quand on examine de près les énoncés scientifiques et techniques, on constate que leur structure syntaxique n'est pas plus compliquée que celle des énoncés non techniques dont tout lecteur (dans le cas de l'écrit) et tout auditeur (dans le cas de l'oral) ont l'habitude. En fait, les énoncés scientifiques et techniques étant essentiellement informatifs et plutôt denses, ils font appel à des structures relativement simples et peu variées. En effet, si l'on part de l'hypothèse que l'auteur élabore son énoncé de manière à être compris par ses destinataires, ce qui est par définition le cas dans l'énoncé informatif (encore qu'on puisse douter du caractère universel de la validité sociologique de ce principe dans les milieux scientifiques), il est compréhensible, voire nécessaire, qu'il simpli- 
fie la structure de son énoncé pour compenser l'effort demandé au récepteur du fait de sa densité informationnelle. Or, dans les langues européennes, la structure syntaxique de l'énoncé reflète de manière assez claire les relations fonctionnelles et logiques entre les référents désignés par les termes techniques, et ce par le biais des déclinaisons, conjugaisons et mots grammaticaux qui indiquent le temps, le sujet, l'objet, la nature du complément, les liens de coordination et de subordination entre les groupes nominaux, qui, avec les verbes, portent l'essentiel de l'information. La structure syntaxique de la phrase permet donc, dans ces langues européennes, de dégager assez clairement la structure logique et fonctionnelle reliant les référents désignés par des groupes nominaux, qui correspondent à la majorité des termes spécialisés (Rey 1979).

Dans cet énoncé, la majorité des termes appartiennent à la deuxième catégorie : simples ou composés, ils ne comportent que des mots courants ("module", "signatures", "méthodes", "reconnaissance», "formes", "espaces", "observations "), mais ne sont pas réellement «compris" par le non-spécialiste, car ils désignent des référents précis qu'il ne connaît pas.

La transparence de ces termes et la simplicité de la structure de la phrase permettent toutefois au lecteur de comprendre lors d'une deuxième lecture qu'il y est question de "méthodes" "puissantes» mais limitées, que l'énoncé explique les limites et en donne une illustration.

En examinant la phrase plus attentivement, en analysant les relations syntaxiques et les termes, et en s'appuyant sur son bagage cognitif, le non-spécialiste peut élaborer des hypothèses qui lui permettent de construire un modèle du sens de la phrase :

- Les limites des méthodes se manifestent par des " ambiguïtés " qui se traduisent par des "signatures semblables " même quand les " anomalies " sont " différentes". On peut donc imaginer que ces méthodes ont notamment pour mission de détecter des " anomalies" de divers types, que ces anomalies se voient à travers des "signatures" assimilées à des "formes ", et que les " limites" se réfèrent à l'incapacité des "méthodes" de distinguer entre elles des " anomalies» différentes. Pour visualiser l'idée, le lecteur peut imaginer un modèle mental où il est question d'anomalies dans la structure de métaux, visibles à travers des formes précises (les " signatures ") se révélant au microscope et pouvant être analysées par des "méthodes " automatiques de reconnaissance des formes.

- De même, le sens général de "module " étant connu par le non-spécialiste, le lecteur peut imaginer que ces méthodes de reconnaissance des formes font appel à des ensembles électroniques séparés (les "modules") dont chacun compare la forme avec une forme étalon représentée par un "modèle physique" tel qu'un rectangle, un triangle, etc., les informations traitées directement par le "modèle physique » étant par exemple le nombre et la valeur des angles dans la forme analysée.

- Enfin, les « espaces d'observation " peuvent être imaginés comme la définition abstraite de ce que l'on peut " observer " par ces méthodes, et les " dimensions restreintes " peuvent désigner les limites de ce champ d'observation.

Le lecteur armé d'une culture technologique et mathématique plus solide élaborera peut-être un modèle plus proche de la réalité; l'association "espace »-« dimensions ", notamment, évoquera probablement pour lui les espaces vectoriels. Le lecteur non spécialiste pourra d'ailleurs lui aussi améliorer son modèle à mesure qu'il assimilera des informations supplémentaires dans la suite de l'énoncé. Toutefois, avec le modèle très rudimentaire présenté plus haut, il dispose déjà d'informations non triviales et a une certaine capacité d'anticipation. Il peut s'attendre par exemple à ce que l'on envisage d'améliorer les méthodes en dotant les modules de modèles physiques plus complets, ou que l'on remplace ces modules par d'autres, plus perfectionnés ; il 
comprendra le lien logique entre l'énoncé et d'éventuels commentaires portant sur la recherche de nouvelles méthodes, sur le coût des ambiguïtés non résolues, etc. Le degré de compréhension pouvant être atteint par un lecteur non spécialiste lui permet donc de suivre l'argumentation de l'auteur dans ses grandes lignes.

\section{L'analyse lexicale}

La " compréhension" de l'énoncé expliquée ci-dessus peut d'ailleurs être améliorée sensiblement par le lecteur non spécialiste : en effet, en règle générale, les termes de la deuxième catégorie (qui ont une certaine transparence) et de la troisème catégorie (totalement incompréhensibles) peuvent être rapprochés de la première catégorie (les termes compris) par une analyse qui peut s'effectuer à trois niveaux.

1. L'analyse morphologique : les préfixes, suffixes, composants connus du terme, désinences et autres caractéristiques du terme livrent des indications sur son sens. La chose est évidente dans les termes composés tels que "méthodes de reconnaissance de formes " et " espaces d'observation ", mais elle se retrouve aussi dans l'analyse de termes non composés tels que " céphalopode " (on reconnaît " tête " dans " céphalo " et "pied" dans "pode ") ou " ondographe " (on y reconnaît " onde » et " graphe» — voir aussi, à propos de l'analyse morphologique des termes japonais (Gile 1984a).

2. L'analyse contextuelle : en contexte, les différents termes sont liés par des relations logiques et fonctionnelles à d'autres termes, et ces relations apportent elles aussi des indications sur le sens des termes inconnus. L'analyse de l'exemple précédent, présentée ci-dessus, illustre ce type d'analyse.

3. La recherche extérieure : le lecteur peut également faire appel à des sources extérieures - spécialistes, dictionnaires, manuels ou revues, spécialisés ou de vulgarisation, etc. (pour un traitement plus détaillé de la recherche extérieure, voir Gile 1984c).

L'analyse lexicale permet donc de ramener un énoncé scientifique et technique apparemment impénétrable au premier abord à un énoncé dont la compréhension " modélisée " permet au lecteur non spécialisé de :

- retirer des informations non triviales

- suivre le raisonnement de l'auteur.

\section{LES BESOINS DU TRADUCTEUR}

En réalité, une telle analyse de l'énoncé n'est pas " naturelle ": dans la pratique, les textes sont adressés à des destinataires qui ont le bagage cognitif et les connaissances terminologiques nécessaires à la compréhension. La traduction représente un phénomème unique dans la communication : le relais humain qu'est le traducteur doit restituer intégralement un message alors qu'il n'est équipé ni pour le recevoir ni pour le transmettre, étant donné son infériorité cognitive et terminologique par rapport à l'émetteur et au récepteur. C'est pourquoi l'analyse lui est indispensable pour compenser ses lacunes (voir à ce sujet Gile 1984a).

Toutefois, en général, le niveau de compréhension nécessaire à la traduction n'est pas identique à celui des destinataires. En fait, s'il est toujours avantageux pour le traducteur d'approfondir au maximum sa compréhension des textes qu'il traduit, ne seraitce qu'en raison du bagage cognitif complémentaire que cette compréhension lui fait acquérir et qui est susceptible de lui servir par la suite, ses besoins ponctuels peuvent être définis avec une certaine précision :

- En général, les relations logiques et fonctionnelles entre les différents termes spécialisés doivent être clairement identifiées afin que le traducteur puisse remanier librement la structure de l'énoncé de manière à rédiger une traduction claire et lisible, donc conforme au génie de la langue d'arrivée. Il existe certaines langues d'arrivée dont le génie favorise 1'ellipse (le japonais en est un exemple — Gile 1984a) ; dans ces lan- 
gues, les relations entre les éléments n'étant pas toujours précisées, leur compréhension n'est pas toujours indispensable aux strictes fins de la traduction. Ce cas représente cependant l'exception plutôt que la régle.

- Du point de vue terminologique, trois cas de figure fondamentaux peuvent se présenter :

a) Le traducteur trouve un terme en langue d'arrivée dont il est sûr qu'il correspond au terme en langue de départ dans le contexte de la traduction : le cas se présente dans des traductions en langage très codifié, ou dans des situations où une certaine normalisation terminologique est acquise, ou encore quand le contexte est suffisamment précis pour que la relation d'" équivalence " contextuelle entre le terme en langue de départ et un terme apparaissant dans un document de référence fiable en langue d'arrivée puisse être établie avec certitude. Dans ce premier cas de figure, le transcodage terminologique est possible sans la compréhension du terme.

b) Le traducteur doit choisir entre deux ou plusieurs termes en langue d'arrivée pour restituer le terme en langue de départ : c'est notamment le cas quand le dictionnaire bilingue consulté propose plusieurs " équivalents " possibles et qu'il faut en sélectionner un seul. Dans ces conditions, le traducteur doit avoir suffisamment d'indications sur le sens du terme en langue de départ et sur le sens de chacun des termes proposés en langue d'arrivée pour éliminer tous les candidats non acceptables. Le degré de compréhension requis dans ce cas varie selon les circonstances, et notamment en fonction de la distance sémantique entre les différents "équivalents" proposés : pour faire un choix entre "back-fill " et "cluster " comme " équivalents" possibles de "entassement ", il suffit de comprendre d'après le contexte qu'on se situe dans le bâtiment ou dans la cristallographie, mais le choix entre "carrier", "catch", "driver » et "dog " pour " entraîneur " dans le domaine de la mécanique demande une certaine précision dans la compréhension du sens de chacun de ces termes. Ce choix nécessite d'ailleurs souvent, outre la compréhension des termes, des connaissances sur les usages terminologiques dans le milieu auquel appartient l'auteur et dans le milieu des destinataires.

c) Le traducteur ne connaît pas d'" équivalents " possibles et doit rendre le terme par ses propres moyens linguistiques et lexicologiques : le cas se présente quand il n'existe pas d'" équivalence » terminologique entre la langue de départ et la langue d'arrivée, ou quand le traducteur ne dispose pas des sources et outils terminologiques lui permettant de la trouver. Parfois, le traducteur peut se tirer d'affaire par une paraphrase ou une autre désignation indirecte du référent qui permet au destinataire de comprendre le message aussi bien que si le terme précis avait été employé (voir en ce qui concerne l'interprétation, Gile 1984b). Dans d'autres cas, il doit décrire ou définir avec une certaine précision le référent du terme original ou créer un nouveau terme en langue d'arrivée. Dans ce troisième cas de figure, la compréhension du traducteur doit parfois se rapprocher de celle du spécialiste.

\section{LA "COMPRÉHENSION FLOTTANTE " ET LES QUALITÉS INTELLECTUELLES DU TRADUCTEUR}

L'analyse de l'énoncé par le traducteur débouche sur un " modèle flottant » de son sens. Ce modèle se compose d'une structure logique et fonctionnelle qui relie des éléments (les référents des différents termes) plus ou moins proches d'une réalité connue du traducteur; le modèle "flotte" donc au-dessus de la réalité, avec pour seuls points d'appui concrets les termes de la première catégorie, dont le référent est bien connu du traducteur. Ce " modèle flottant » est dans une certaine mesure comparable à la compréhension que l'on a des instructions d'un passant à qui on demande son chemin : on "comprend » un certain cheminement (aller tout droit jusqu'à la $2^{2}$ rue à droite, la prendre, puis tourner à gauche devant la boulangerie, etc.) et on a un point d'appui concret 
(l'endroit où l'on est) ainsi que des points d'appui semi-concrets (on sait à peu près à quoi ressemble une boulangerie), mais le modèle mental de l'explication reste "flottant" tant qu'on n'a pas parcouru le chemin et tant que les instructions abstraites n'ont pas été remplacées dans l'esprit par la connaissance réelle du parcours.

À première vue, ce "modèle flottant » est rare dans la vie quotidienne, puisqu'on est en général destinataire des énoncés que l'on essaie de comprendre, et que l'on connaît bien tous les termes employés. La structure logique de l'énoncé est donc fermement reliée à la réalité par ces termes de la première catégorie.

Et pourtant, sait-on précisément ce que sont les " antibiotiques " que l'on prend pour combattre une infection, à quoi correspondent les quatre chevaux fiscaux d'une petite voiture, quels sont les éléments pris en compte dans le calcul du produit national brut d'un pays ? Ce sont en réalité des mots de la deuxième catégorie, car on sait que les antibiotiques " tuent les microbes", qu'une voiture de quatre chevaux est moins puissante qu'une voiture de dix chevaux, et que plus le PNB d'un pays est élevé, plus ce pays est " riche ", mais on ne " comprend " pas ces termes comme un biologiste, un ingénieur mécanicien, un économiste. Le non-spécialiste peut pourtant suivre une argumentation où ils figurent, voire s'en servir pour présenter ses propres idées - en fait, il a de l'énoncé une " compréhension flottante " analogue à la compréhension flottante du traducteur.

La «compréhension flottante » du traducteur est essentiellement la même que la « compréhension flottante » du lecteur et de l'auditeur dans la vie courante, mais en diffère par des paramètres quantitatifs et par les circonstances qui l'entourent :

- Dans la traduction scientifique et technique, la " compréhension flottante " intervient très souvent, alors qu'elle est plutôt rare dans la vie courante.

- Dans la traduction scientifique et technique, la proportion des termes de la deuxième et de la troisième catégorie dans l'énoncé est importante, alors qu'elle est faible dans la vie quotidienne. $\mathbf{L a}$ " compréhension flottante " du traducteur " flotte " donc plus loin de la "réalité » concrète que celle du lecteur ou de l'auditeur ordinaire.

- Comme il a été expliqué plus haut, le traducteur scientifique et technique a l'obligation professionnelle d'arriver à un certain degré de compréhension de l'énoncé dans son ensemble, afin de pouvoir le restituer efficacement dans la langue d'arrivée. Le lecteur ou l'auditeur ordinaire se concentre sur ce qui l'intéresse et peut laisser de côté les éléments de l'énoncé qui lui semblent confus ou peu importants.

La " compréhension flottante " est l'aboutissement d'un processus d'analyse ; le traducteur polyvalent et l'interprète, qui tous les deux ont affaire à des énoncés spécialisés dans des domaines qu'ils ne connaissent pas, doivent non seulement avoir une bonne maitrise de leurs langues de travail et savoir rédiger (ou s'exprimer oralement) dans leur langue d'arrivée, mais aussi avoir l'esprit logique et une réelle faculté d'abstraction, et ce pour pouvoir se servir de ces modèles flottants tant pour restituer le message sous une forme efficace et conforme au génie de la langue d'arrivée que pour renforcer leur propre bagage cognitif à mesure que l'énoncé leur apporte des informations supplémentaires. C'est pourquoi en général, les écoles de traduction reconnues par les organisations internationales et les associations de traducteurs sont des établissements universitaires. En effet, la formation à la traduction s'accompagne d'un perfectionnement linguistique et d'une acquisition de connaissances, mais elle se fonde surtout sur une discipline intellectuelle où l'analyse est reine. 


\section{RÉFÉRENCES}

FOLKART, Barbara (1984) : "A Thing-bound Approach to the Practice and Teaching of Technical Translation ", META, 29-3, septembre.

GILE, Daniel (1984a) : "L'enseignement de la traduction japonais-français : une formation à l'analyse ", compte-rendu du Colloque sur la communication entre langues européennes et langues orientales tenu à Montvillargenne les 23, 24, 25 janvier 1984 (sous les auspices de la Commission des communautés européennes - DG XIII).

GILE, Daniel (1984b) : "Des difficultés de la transmission informationnelle en interprétation simultanée ", Babel, XXX-1, janvier.

GILE, Daniel (1984c) : "La recherche terminologique dans la traduction scientifique et technique japonaisfrançais : une synthèse ", META, 29-3, septembre.

HERBERT, Jean (1952) : Manuel de l'interprète, Librairie de l'Université, Genève, Georg.

KOURGANOFF, Vladimir (1980) : "Quelques traquenards du thème scientifique anglais ", Traduire, $\mathrm{n}^{\circ}$ 103, juin.

MATHE, G., G. RICHET, J.-P. BENHAMOU, P. BERTHAUX, J.-P. HARDOUIN, et J. LISSAC (1965) : Sémiologie médicale, t. 2, Paris, Flammarion.

PAPIN, B., P. BERNARD, C. CHAOUIYA, et J.-L. ARTAUD (1984) : "Surveillance des réacteurs à eau sous pression par analyse de processus", Actes du Colloque international d'intelligence artificielle, Marseille, 24-27 octobre.

REY, Alain (1979) : la Terminologie, noms et notions, Paris, PUF.

SÉLESKOVITCH, Danica (1968) : l'Interprète dans les conférences internationales, Paris, Minard Lettres modernes. 